\title{
CONTRACTIVE PROJECTIONS IN NONATOMIC FUNCTION SPACES
}

\author{
BEATA RANDRIANANTOANINA
}

(Communicated by Dale Alspach)

\begin{abstract}
We prove that there are no 1-complemented subspaces of finite codimension in separable rearrangement-invariant nonatomic function spaces not isometric to $L_{2}$.
\end{abstract}

We study contractive projections onto finite-codimensional subspaces of real nonatomic function spaces. In general such projections are not common. It is well known that only in Hilbert space there exists a contractive projection onto every subspace of fixed finite codimension (cf. [1]).

The study of contractive projections (and more general projections with minimal norm) is important in approximation theory (cf. the survey of Cheney and Price [3]).

It is known that there are no 1-complemented subspaces of finite-codimension in $C[0,1]$ (Wulbert [13]) and in $L_{1}(\mu)$ if the measure $\mu$ is nonatomic ([6, Corollary IV.1.15], [4]). De Figueiredo and Karlovitz [5] (1970) proved that if $\mu$ is nonatomic, then there are no 1-complemented hyperplanes in $L_{p}(\Omega, \mu)$, $1<p<\infty, p \neq 2$ (cf. also [2]).

In this paper we prove that in rearrangement-invariant nonatomic function spaces not isometric to $L_{2}$ there are no 1-complemented subspaces of any finite codimension.

We use the terminology and notation as in [9].

Our method of proof is surprisingly simple-it is based on the following observation:

Proposition 1 (cf. [7, 11]). In a real Banach space $X$ if $P$ is a projection, then $\|I-P\|=1$ (where I denotes identity operator) if and only if $x^{*}(P x) \geq 0$ for all $x \in X$ and $x^{*} \in X^{*}$ norming for $x$.

In [7, Theorem 4.3] (cf. [8, 10]) Kalton and the author proved the nonexistence of 1-complemented hyperplanes in a wide class of nonatomic function spaces. However, the original theorem uses special technical phraseology, so we state it below in the language of projections:

Received by the editors September 22, 1993; the contents of this paper were presented at the conference "Algebras in Analysis" at Kent State University, Kent, Ohio, September 1993.

1991 Mathematics Subject Classification. Primary 46B20, 46B04, 46E30. 
Theorem 2. Suppose $X$ is a real order-continuous Köthe function space on $(\Omega, \mu)$ and $\mu$ is nonatomic. Then the hyperplane $H$ in $X$ is 1-complemented if and only if there exists a nonnegative measurable function $\omega$ with $\operatorname{supp} w=$ $B=\operatorname{supp} f$, where $f \in H^{\perp} \subset X^{*}$, so that for any $x \in X$ with $\operatorname{supp} x \subset B$

$$
\|x\|=\left(\int|x|^{2} w d \mu\right)^{1 / 2}
$$

Hence there are no 1-complemented hyperplanes in $X$ unless $L_{2}$ is isometric to a band in $X$. In particular, there are no 1-complemented hyperplanes in separable r.i. spaces on $[0,1]$ [7, Theorem 4.4].

As part of the proof of Theorem 2 we proved the following fact, which we state separately for the future use.

Proposition 3 ([10, Proposition 2.8]). Let $X$ be an order-continuous Köthe function space on $(\Omega, \mu)$, where $\mu$ is nonatomic. Suppose that the set

$$
\Lambda=\left\{\frac{x^{*}}{x}: x \in X, x^{*} \in X^{*} \text { norming for } x\right\}
$$

is one-dimensional, i.e., $\Lambda \subset\{a w: a \in \mathbb{R}\}$ for some $w \in L_{0}(\Omega, \mu)$. Then $X$ is isometric to $L_{2}(w d \mu)$.

Now we are ready to prove our main result.

Theorem 4. Suppose $\mu$ is nonatomic and $X$ is a separable r.i. space on $([0,1], \mu)$ not isometric to $L_{2}$. Then there are no 1-complemented subspaces of any finite codimension in $X$.

For the proof we need the following measure-theoretic lemma.

Lemma 5. Suppose $\mu$ is nonatomic, and suppose $f_{1}, \ldots, f_{n}, g_{1}, \ldots, g_{n} \in$ $L_{1}(\mu)$ are such that $g_{1}, \ldots, g_{n}$ are linearly independent and

$$
\sum_{i=1}^{n}\left(\int h f_{j} d \mu\right)\left(\int h g_{j} d \mu\right) \geq 0
$$

whenever $|h|=1$ a.e. Then $\left\{f_{j}\right\}_{j=1}^{n} \subset \operatorname{span}\left\{g_{j}\right\}_{j=1}^{n}$.

Proof. Consider the operator $T: \mathbb{R}^{2 n} \rightarrow L_{1}(\mu)$ defined by

$$
T\left(a_{1}, b_{1}, \ldots, a_{n}, b_{n}\right)=\sum_{i=1}^{n} a_{i} f_{i}+b_{i} g_{i} .
$$

To prove the lemma it is enough to show that the dimension of the range of $T$ is equal to $n$, since $f_{1}, \ldots, f_{n}, g_{1}, \ldots, g_{n}$ are contained in the range and $g_{1}, \ldots, g_{n}$ are linearly independent. For that it is enough to show that $\operatorname{dim} T^{*}\left(L_{\infty}(\mu)\right) \leq n$. Notice that the operator $T^{*}$ is defined by $T^{*} h=$ $\left(\int h f_{1} d \mu, \int h g_{1} d \mu, \ldots, \int h f_{n} d \mu, \int h g_{n} d \mu\right)$ for $h \in L_{\infty}(\mu)$, and let $\Gamma=$ $\left\{T^{*} h:|h|=1\right.$ a.e. $\}$.

Clearly $T^{*}\left(L_{\infty}(\mu)\right)=\operatorname{span} \Gamma$, and we immediately see that $\Gamma=-\Gamma$. Now consider the $\mathbb{R}^{2 n}$-valued measure $\bar{\mu}: \bar{\mu}(A)=\left(\int \chi_{A} f_{1} d \mu, \int \chi_{A} g_{1} d \mu, \ldots\right.$, $\left.\int \chi_{A} f_{n} d \mu, \int \chi_{A} g_{n} d \mu\right)$. By Liapunoff's theorem [12] the angle $\mathscr{R}(\bar{\mu})$ of $\bar{\mu}$ is 
convex and thus also $\Gamma$ is convex $\left(\Gamma=T^{*} 1-2 \mathscr{R}(\bar{\mu})\right)$. Therefore inequality (1) yields that

$$
\sum_{j=1}^{n} s_{j} t_{j} \geq 0 \quad \text { for every }\left(s_{1}, t_{1}, \ldots, s_{n}, t_{n}\right) \in T^{*}\left(L_{\infty}(\mu)\right) .
$$

To finish the proof consider $V=\operatorname{span}\left\{e_{2 k-1}-e_{2 k}: k=1, \ldots, n\right\} \subset \mathbb{R}^{2 n}$, where $e_{j}$ denotes the natural basis of $\mathbb{R}^{2 n}$. By (2) $V \cap T^{*}\left(L_{\infty}(\mu)\right)=\{0\}$, so $\operatorname{dim} T^{*}\left(L_{\infty}(\mu)\right) \leq n$.

Proof of Theorem 4. Suppose that $F$ is a closed linear subspace of codimension $n$ in $X$, and let $u_{1}, \ldots, u_{n}$ be linearly independent functions in $X$ such that $\operatorname{span}\left\{F, u_{1}, \ldots, u_{n}\right\}=X$. Denote $P: X \rightarrow F$ a contractive projection onto $F$, and consider $Q=I-P$. Then $Q=\sum_{j=1}^{n} f_{j} \otimes u_{j}$ for some linearly independent $f_{1}, \ldots, f_{n} \in X^{*}$.

By Proposition 1 for any $x \in X$ and $x^{*} \in X^{*}$ norming for $x^{*}(Q x) \geq 0$. Next for any $h$ with $|h|=1$ a.e. $h x^{*}$ is norming for $h x$ if $x^{*}$ is norming for $x$. Hence $h x^{*}(Q(h x)) \geq 0$, i.e.,

$$
\sum_{j=1}^{n}\left(\int f_{j} h x d \mu\right)\left(\int u_{j} h x^{*} d \mu\right) \geq 0 .
$$

By Lemma $5 f_{1} x \in \operatorname{span}\left\{u_{j} x^{*}: j=1, \ldots, n\right\}$. So if $B=\operatorname{supp} f_{1}(\mu(B)>$ $0)$, then $\left.\frac{x}{x^{*}}\right|_{B} \in \operatorname{span}\left\{\frac{u_{j}}{f_{1}}: j=1, \ldots, n\right\}$. By re-arrangement invariance of $X$ for every measure-preserving map $\sigma:[0,1] \rightarrow[0,1] x^{*} \circ \sigma$ is norming for $x \circ \sigma$ and so

$$
\left.\frac{x \circ \sigma}{x^{*} \circ \sigma}\right|_{B} \in \operatorname{span}\left\{\frac{u_{j}}{f_{1}}: j=1, \ldots, n\right\},
$$

i.e., the set $\left\{\left.\left[\left(\frac{x}{x^{*}}\right) \circ \sigma\right]\right|_{B} \mid \sigma:[0,1] \rightarrow[0,1]\right.$ measure preserving $\}$ is finite dimensional, which is impossible unless $\frac{x}{x^{*}}$ is a constant. But then by Proposition 3 we conclude that $X$ is isometric to $L_{2}[0,1]$, contrary to our assumption.

Remark. Notice that in the proof of Theorem 4 we use re-arrangement invariance of $X$ only in the final step to conclude that if the set $\left\{\frac{x}{x^{*}}: x \in X, x^{*} \in X^{*}\right.$ norming for $x\}$ is finite-dimensional, then it is one-dimensional. A similar conclusion is true also in spaces of the form $X(Y), X_{1}\left(X_{2}\left(\ldots\left(X_{m}\right) \ldots\right)\right)$, where $X$, $Y, X_{j}$ are r.i. and Theorem 4 holds as stated also for those spaces.

\section{ACKNOWLEDGMENTS}

I wish to express my gratitude to Professor Nigel Kalton for his interest in this work and many valuable discussions. I also would like to thank Professor Alspach for suggesting an elegant simple approach to the proof of Lemma 5.

\section{REFERENCES}

1. D. Amir, Characterizations of inner product spaces, Birkhäuser Verlag, Basel, Boston, and Stuttgart, 1986.

2. B. Beauzamy and B. Maurey, Points minimaux et ensembles optimaux dans les espaces de Banach, J. Funct. Anal. 24 (1977), 107-139. 
3. E. W. Cheney and K. H. Price, Minimal projections, Approximation Theory (A. Talbot, ed.) (Proceedings of a symposium held in Lancaster, July 1969), Academic Press, New York, 1970, pp. 261-289.

4. R. G. Douglas, Contractive projections on an $L_{1}$ space, Pacific J. Math. 15 (1965), 443-462.

5. D. G. de Figueiredo and L. A. Karlovitz, On the extension of contractions of normed spaces, Nonlinear Functional Analysis, Proc. Sympos. Pure Math., vol. 18, Part 1, Amer. Math. Soc., Providence, RI, 1970, pp. 95-104.

6. P. Harmand, D. Werner, and W. Werner, M-ideals in Banach spaces and Banach algebras, Lecture Notes in Math., vol. 1547, Springer, Berlin, Heidelberg, and New York, 1993.

7. N. J. Kalton and B. Randrianantoanina, Surjective isometries of rearrangement-invariant spaces, Quart. J. Math. Oxford (to appear).

8. $ـ$, Isometries of rearrangement-invariant spaces, C. R. Acad. Sci. Paris Sér. I Math. 316 (1993), 351-355.

9. J. Lindenstraus and L. Tzafriri, Classical Banach spaces, vol. 2, Function spaces, SpringerVerlag, Berlin, Heidelberg, and New York, 1979.

10. B. Randrianantoanina, Isometries of function spaces, Ph.D. Dissertation, University of Missouri-Columbia, 1993.

11. H. P. Rosenthal, Contractively complemented subspaces of Banach spaces with reverse monotone (transfinite) bases, Longhorn Notes, The University of Texas Functional Analysis Seminar, 1984-85, pp. 1-14.

12. W. Rudin, Functional analysis, 2nd ed., McGraw-Hill, New York, 1991.

13. D. E. Wulbert, Projections of norm 1 on $C(X)$, Notices Amer. Math. Soc. 15 (1968), 362. 43403

Department of Mathematics, Bowling Green State University, Bowling Green, Ohio

Current address: Department of Mathematics, The University of Texas, Austin, Texas 78712

E-mail address: brandri Omath.utexas.edu 